\title{
Neuropsychiatric Manifestations of Cognitively Advanced Idiopathic Normal Pressure Hydrocephalus
}

\author{
Robert Mathew $^{\mathrm{a}, \mathrm{d}}$ Sauda Pavithran ${ }^{\mathrm{b}} \quad$ P. Byju ${ }^{\mathrm{c}}$ \\ a Sree Mookambika Institute for Medical Sciences, Padanilam, Kanyakumari District, \\ Kulasekharam, India; ${ }^{b}$ Department of Neurology, SK Hospital, Trivandrum, India; \\ 'Department of Neurology, Pushpagiri Institute for Medical Sciences, Thiruvalla, India; \\ ${ }^{\mathrm{d}}$ Anugraham Neurocare, Pattom, Trivandrum, India
}

\author{
Keywords \\ Behavioral and psychological symptoms of dementia · Cognition · Dementia · \\ Hydrocephalus · Neuropsychiatry · Normal pressure hydrocephalus · Subcortical dementia
}

\begin{abstract}
Background: Neuropsychiatric manifestations of patients with idiopathic normal pressure hydrocephalus (iNPH) have not been studied in a systematic way. Aim: To study the spectrum of neuropsychiatric abnormalities in patients with iNPH. Patient Selection and Evaluation: Patients attending 3 different tertiary care centers during three consecutive time periods spanning from 2010 to 2015 were analyzed for neuropsychiatric manifestations. Patients diagnosed as having probable or possible iNPH as per the consensus criteria were included in the study. Neuropsychiatric manifestations were captured by a comprehensive inventory (Cambridge Behavioral Inventory, CBI). Results: The CBI score was available for 41 patients. The mean Mini-Mental State Examination score was 15.37 (SD 7.2) and the Addenbrooke's Cognitive Examination score was 34.95 (SD 19.67), thereby indicating cognitively advanced iNPH. All patients had impairment in one or more items on the CBI. The mean score was 55.46 (SD 27) out of 180, thereby indicating a mild degree of impairment. Among the subscores, impairment with motivation was the most observed abnormality followed by memory impairment. When the $\mathrm{CBI}$ total score and subscores were compared, all of them (except motivation) were higher for Alzheimer's disease; however, none was statistically significant. Even though the motivation score was higher for $\mathrm{NPH}$, the difference did not reach statistical significance. Conclusions: It can be concluded that neurobehavioral abnormalities are common in patients with cognitively advanced normal pressure hydrocephalus. However, the intensity of involvement appeared less when compared to Alzheimer's disease. Apathy appears to be the most common impairment.




\section{Introduction}

Neuropsychiatric symptoms can induce marked disability in patients with dementia and increase caregiver distress and can be observed in up to $90 \%$ of patients with dementia [1, 2]. Initially, it was thought that neuropsychiatric manifestations are features unique to advanced dementia. But now we know that these symptoms can manifest at a very early stage and even in patients with minimal cognitive impairment [3].

In dementia patients, neuropsychiatric manifestations affect the quality of life and contribute to the caregiver burden. Neuropsychiatric manifestations have been studied extensively in patient with Alzheimer's disease (AD) and the commonest is apathy and depression. As the disease progresses, aggression, delusions, and hallucinations become more common. Studies on neuropsychiatric manifestations of idiopathic normal pressure hydrocephalus (iNPH) are few [4-6]. The available studies are either restricted in patient number or consist mostly of underevaluated patients. One of the recent studies on cognition in NPH did not address neuropsychiatric manifestations [7]. A proper understanding of the neuropsychiatric manifestations will shed more light into the neurobiology of the disease. Furthermore, this gives an opportunity to develop more targeted drug treatments as well as nonpharmacological management techniques to enhance caregiver coping skills.

In our study, our postulation was that the majority of patients would have some type of neuropsychiatric impairment as we were studying cognitively advanced iNPH patients. One of the recent studies on cognition in iNPH showed that in cognitively advanced iNPH neuropsychologic impairment was similar to AD [7].

Hence, in this study, we expected cognitively advanced patients to have neuropsychiatric manifestations similar to patients with advanced AD. The aim was to study the spectrum of neuropsychiatric abnormalities in patients with iNPH.

\section{Patient Selection and Evaluation}

It was a prospective observational cross-sectional study. Patients diagnosed as having probable or possible iNPH as per the consensus criteria were included in the study. As per the criteria, it was mandatory for all patients to have hydrocephalus on imaging with clinical features of iNPH. Patients who were having coexisting neurological illness of the central nervous system were excluded from the study. Patients who were excluded were those with stroke, space-occupying lesions, and traumatic head injury [8]. AD was defined according to the NINCDS-ADRDA criteria [9].

Patients attending 3 different tertiary care centers during 3 consecutive time periods spanning from 2010 to 2015 were analyzed. The patients included those attending outpatient services as well as admitted patients in various medical and surgical wards either as part of dementia evaluation or for unrelated conditions. Many patients in the surgical wards were first seen by a neurologist as part of delirium evaluation and subsequently diagnosed as having dementia. The usual presenting complaint of the patients admitted for dementia evaluation was memory loss. Even though all the patients had gait abnormality, walking difficulty as a presenting complaint was uncommon. All patients were examined by a neurologist (R.M.). The examination consisted of a semistructured interview of the participant's medical history followed by standard physical and neurological examination. The majority of the patients were diagnosed as having dementia for the first time during assessment.

Cognitive function was evaluated with a test battery that included Addenbrooke's Cognitive Examination (ACE), the Mini-Mental State Examination (MMSE), the Trail Making Test Parts A and B, attention span, story recall, line orientation, the Hospital Anxiety and 
Depression Scale, Activity of Daily Living Scale, IQCODE, and the Rey Auditory Verbal Learning Test. The ACE as well as the Activity of Daily Living Scale were available in adapted and standardized form for the local language. The Cambridge Behavioral Inventory (CBI), the Rey Auditory Verbal Learning Test, the Hospital Anxiety and Depression Scale, and the symptoms checklist was available in the local language. The CBI questionnaire was completed by the caregivers. Gait was evaluated by history of walking difficulty as well as by clinical examination. Urinary incontinence was assessed by self-reporting. Informed consent was obtained from all patients. All patients underwent imaging either in the form of a CT scan or MRI scan.

The CBI was developed to assess neuropsychiatric changes in AD and FTLD. It incorporates questions related to symptoms reported in previous studies of $\mathrm{AD}$ and FTLD and has components from the Neuropsychiatric Inventory (NPI) [10]. It has been found to be of use in capturing neurobehavioral abnormalities in other neurodegenerative diseases as well, and hence it may also have diagnostic value [11]. The abridged version of the CBI was used. This contains 45 items each receiving a scale score of $0-4$. This is useful to assess broad neuropsychiatric domains and everyday functioning in a variety of dementia syndromes. The 10 behavioral abnormalities assessed by the CBI includes: delusions, hallucinations, memory and orientation, everyday skills, self-care, abnormal behavior, mood, eating habits, sleep, stereotypy, motor behavior, and motivation. The items were translated into local language.

The informant was asked if the behavior of the patient represented a change from that before the onset of dementia and if it had been present during the past month. The informant was not screened for cognitive impairment. However, the information was collected from the same informant who gave a history of dementia.

\section{Statistics}

SPSS for Windows version 16 was used. Comparison of means was made using Student's $t$ test. Parametric variables were analyzed using ANOVA and a $p$ value of less than 0.5 was taken as significant. Pearson's correlation was used to study correlation between variables.

\section{Results}

A total of 825 patients were screened positive for dementia. At the end of the complete evaluation, 153 patients were diagnosed as having iNPH and 78 were diagnosed as having AD. The remaining 524 patients could not be included in the study either because they were diagnosed with other dementia syndromes or because proper diagnosis could not be confirmed. All the patients had impaired cognition as well as gait abnormality. $70 \%$ were males. The mean age of the patients was 73.72 years (SD 8.06) and the mean education was 7.57 years (SD 4.63). The mean duration of illness at the time of the assessment was 2.15 years (SD 0.89). Urinary incontinence was seen in 25\% of the patients. The mean MMSE score was 15.37 (SD 7.2) and the ACE score was 34.95 (SD 19.67), thereby indicating cognitively advanced iNPH. The results were compared with 14 patients with AD.

CBI score was available for 41 patients (Table 1). All patients had impairment in one or more items on the CBI. The mean score was 55.46 (SD 27) out of a total score of 180, thereby indicating mild degree of impairment. The lowest total score was 14 and the highest score was 111. The individual scores and subscores were compared in terms of number of patients impaired in each item as well as mean scores per item. The severity of impairment was reflected by the mean score. The majority of patients (87\%) had impairment in item 42 ("showing less interest in doing new things"). Considering the individual scores, the highest mean score was in the item "shows little interest in doing new things" (mean score 2.68, SD 1.4) and the lowest score in the item "finds humor or laughs at things others do not find funny" 
Table 1. CBI mean score and subscores

\begin{tabular}{|c|c|c|c|c|c|c|c|}
\hline & $\begin{array}{l}\text { Patients } \\
\text { with NPH, \% } \\
(n=41)\end{array}$ & $\begin{array}{l}\text { Patients } \\
\text { with } \mathrm{AD}, \% \\
(n=14)\end{array}$ & $\begin{array}{l}\text { ANOVA } \\
p\end{array}$ & $\begin{array}{l}\text { Mean CBI } \\
\text { subscore } \\
(\mathrm{NPH})\end{array}$ & $\begin{array}{l}\text { Percentage of } \\
\text { maximum } \\
\text { mean CBI score }\end{array}$ & $\begin{array}{l}\text { Mean CBI } \\
\text { subscore (AD) }\end{array}$ & $\begin{array}{l}\text { ANOVA } \\
p\end{array}$ \\
\hline \multirow{2}{*}{\multicolumn{8}{|c|}{$\begin{array}{l}\text { Neurobehavioral domains } \\
\text { Cognition }\end{array}$}} \\
\hline & & & & & & & \\
\hline MMSE score & & & & $15.37(7.2)$ & & 18.38 (7.27) & 0.033 \\
\hline ACE score & & & & 34.95 (19.67) & & 38.54 (23.37) & 0.363 \\
\hline Memory and orientation & 85.4 & 75 & & 46.79 & $45.05(25.74)$ & $11.54(11.71)$ & 0.20 \\
\hline Everyday skills & 92.7 & 100 & 0.02 & $7.22(5.41)$ & $40.94(29.9)$ & $10.62(7.3)$ & 0.07 \\
\hline Self-care & 48.8 & 75 & & $4.12(5.37)$ & $27.59(34.26)$ & $5(5.9)$ & 0.62 \\
\hline Abnormal behavior & 70.7 & 100 & & $4.2(4.30)$ & $19.18(17.96)$ & $5.54(4.39)$ & 0.33 \\
\hline Mood & 73.2 & 100 & & $3.22(3.10)$ & $22.29(19.13)$ & $4.38(2.9)$ & 0.24 \\
\hline False beliefs & 48.8 & 50 & & $2.6(3.88)$ & $25.31(35.28)$ & $4.15(5.13)$ & 0.25 \\
\hline Eating habit & 56.1 & 75 & 0.04 & $2.87(4.66)$ & $22.76(31.82)$ & $5.8(5.8)$ & 0.07 \\
\hline Sleep & 85.4 & 75 & & $3.68(2.54)$ & $46.93(32.70)$ & $3.69(3.04)$ & 0.99 \\
\hline \multicolumn{8}{|l|}{ Stereotypy and motor } \\
\hline behavior & 85.4 & 75 & & $4.04(3.72)$ & $27.00(24.57)$ & $5.25(4.63)$ & 0.36 \\
\hline Motivation & 100 & 75 & & $8.51(5.49)$ & $50.36(32.65)$ & $6.5(4.01)$ & 0.24 \\
\hline CBI total score & & & & $55.46(27.02)$ & & 66.67 (36.33) & 0.25 \\
\hline $\begin{array}{l}\text { Comparison of measures } \\
\text { of cognition }\end{array}$ & & & & $\begin{array}{l}\text { Mean score } \\
(\mathrm{NPH})\end{array}$ & & $\begin{array}{l}\text { Mean score } \\
\text { (AD) }\end{array}$ & $\begin{array}{l}\text { ANOVA } \\
p\end{array}$ \\
\hline \multicolumn{8}{|l|}{ Test of cognition } \\
\hline MMSE & & & & $15.37(7.2)$ & & 18.38 (7.27) & 0.033 \\
\hline ACE & & & & 34.95 (19.67) & & $38.54(0.37)$ & 0.363 \\
\hline
\end{tabular}

Figures in parentheses indicate SD.

(mean score 0) (Fig. 1). The most severely impaired items going by mean score included "has poor day-to-day memory," "asks the same questions over and over again," "forgets what day it is," and "shows less enthusiasm for his or her usual interests." The 45 individual scores were classified into 10 subscores. All the patients had abnormalities in at least 3 subscores. Twenty patients (48.8\%) had abnormality in 9 out of 10 CBI subscores. The individual scores and subscores are shown in Table 1. Excluding the memory-related symptoms, it was the motivational subscores that were most involved. Other items with substantial impairment included "sleep is disturbed at night," "sleeps more by day than before," "repeatedly uses the same expression or catch phrase," and "has difficulties writing." Table 1 shows the mean score of each subscores.

For comparison, each subscore was converted to a percentage of the maximum score (Fig. 2). In terms of the number of patients involved, secondary to motivation, it was everyday skills that were most involved (97.7\%).

Among the subscores, impairment of motivation was the most observed abnormality followed by memory impairment. Sleep abnormality was seen in a significant number of patients. Comparing the two sleep-related scores, daytime sleeping was more involved in terms of number of patients ( $32 \mathrm{vs.23}$ ) and severity (mean score $1.71 \mathrm{vs.} 1.98$ ) than "sleep is disturbed at night." These patients were compared with 14 patients with AD.

The mean MMSE score as well as the ACE score were lower for patients with NPH than for patients with $\mathrm{AD}$ and the difference was significant for the MMSE score $(p=0.03)$. In terms of the number of patients having each symptom, significant differences were seen only in eating habits and everyday skills, which was higher in AD. When CBI total score and subscores 


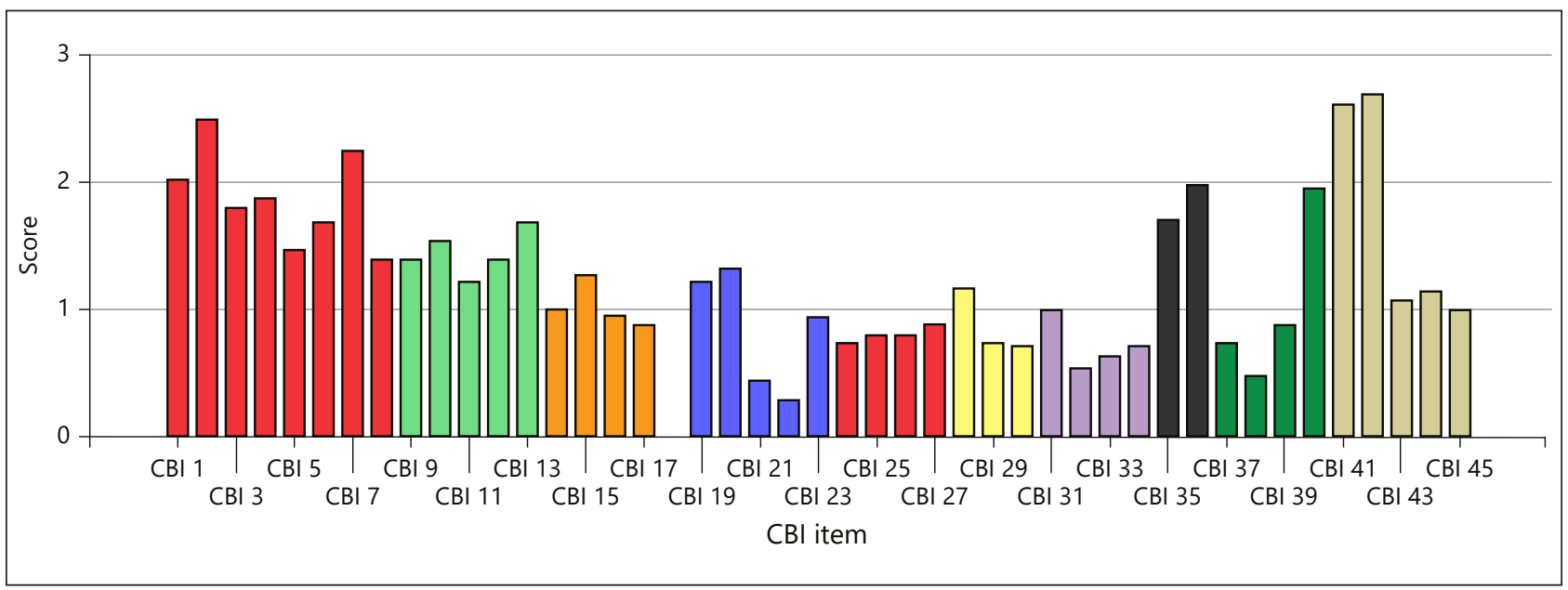

Fig. 1. Mean CBI subscores for each item (i.e., CBI 1-45) corresponding to the number in the inventory. $\mathrm{CBI}$ 1-8: memory; CBI 9-13: everyday skills; CBI 14-17: self-care; CBI 18-23: abnormal behavior: CBI 24-27: abnormal mood; CBI 28-30: abnormal belief; CBI 31-34: abnormal eating habits; CBI 35-36: sleep abnormalities; CBI 37-40: stereotypy and motor behaviors; CBI 41-45: abnormal motivation.

Fig. 2. The mean percentage of the maximum score across each subcategory.

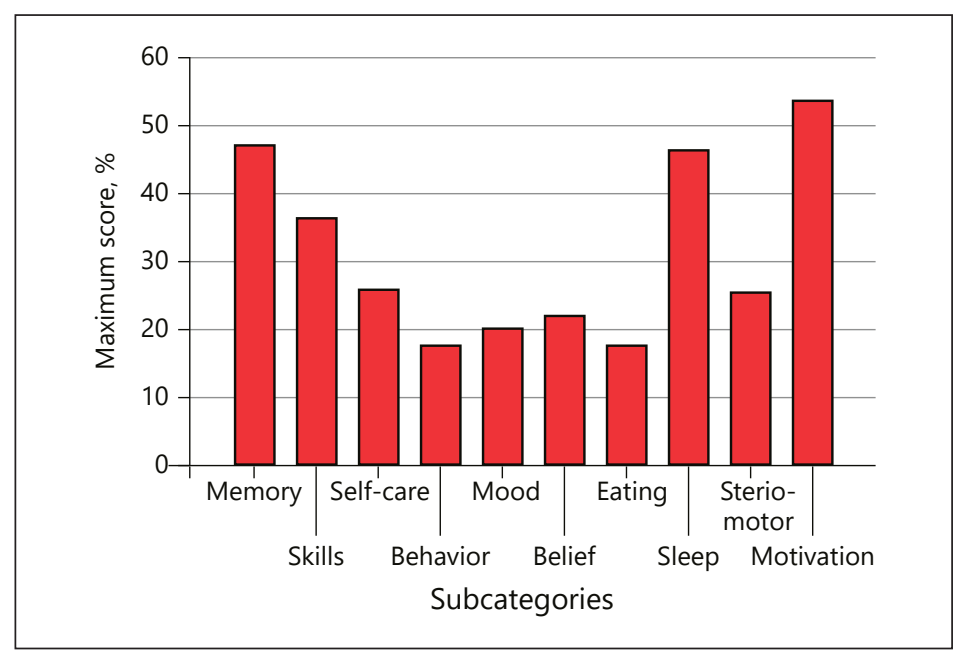

were compared, all the scores except motivation were higher for AD patients; however, none was statistically significant. Even though the motivation score was higher for iNPH patients, the difference did not reach statistical significance.

Correlation of the CBI total score and subscores with the ACE total score and subscores was considered. A significant correlation between the CBI total score and the ACE total score was seen ( $p=0.004$, Pearson's correlation). However, when compared for memory subscores (CBI and ACE), no significant change was seen (partial correlation, $p=0.079$ ). No significant correlation was seen between cognitive subscores and neuropsychiatry subcores. No correlation was seen between duration of illness at the time of presentation and frequency of occurrence of neurobehavioral abnormalities or severity considering the CBI scores.

Three patients presented as having psychiatric illness (not as having dementia). They had prominent delusion, hallucinations, and features of bipolar illness. One of these patients had bipolar illness presenting symptoms at the age of 45 years. She underwent ventriculoperitoneal shunt twice and revision of the shunt once in a span of 15 years. Each time she 
Table 2. Percentage of patients having each domain impaired compared with the present study

\begin{tabular}{|c|c|c|}
\hline \multirow[t]{2}{*}{ Neurobehavioral domains } & \multicolumn{2}{|l|}{ Patients, $\%$} \\
\hline & $\begin{array}{l}\text { present study } \\
(n=41)\end{array}$ & $\begin{array}{l}\text { Kito et al. [6] } \\
(n=38)\end{array}$ \\
\hline Memory and orientation & 85.4 & \\
\hline Everyday skills & 92.7 & \\
\hline Self-care & 48.8 & \\
\hline Abnormal behavior & 70.7 & $\begin{array}{l}17.2 \\
\text { (agitation) } \\
12.2 \\
\text { (disinhibition) }\end{array}$ \\
\hline Mood & 73.2 & $\begin{array}{l}14.1 \\
\text { (depression) } \\
10.8 \\
\text { (irritability) }\end{array}$ \\
\hline False belief & 48.8 & 17 \\
\hline Eating habit & 56.1 & \\
\hline Sleep & 85.4 & \\
\hline Stereotypy and motor behavior & 85.4 & 14.1 \\
\hline Motivation & 100 & $\begin{array}{l}70 \\
\text { (apathy) }\end{array}$ \\
\hline Cognition (MMSE) & 15 & 19 \\
\hline
\end{tabular}

presented with psychotic features which went into remission after shunt surgery only to reappear again after a few years corresponding to worsening of the hydrocephalus.

We used the CBI to assess neuropsychiatric abnormalities. Studies using the CBI were not available for comparison. However, our results were compared with the study of Kito et al. [6] in which the NPI was used (Table 2). In the cohort of Kito et al. [6], the patients were cognitively less impaired when compared to the present study and the percentage of patients having various behavioral abnormalities was lower. The abnormality in the majority of patients in that study was apathy (70\%) as opposed to motivation abnormality $(100 \%)$ in the present study. We found that the results are comparable within the limits.

\section{Discussion}

Neurobehavioral symptoms in dementia can be defined as signs and symptoms of disturbed perception, thought, mood or behavior. They are the most complex, stressful and costly aspect of dementia care [12]. More than cognitive impairment, it is these symptoms that lead to health care problems, poor patient health and income loss for family and caregivers. The causes for these behaviors may be many disease-related factors, caregiver factors, and environment triggers [13].

To identify neuropsychiatric abnormalities, it is important to use a valid easy-to-use instrument, such as the CBI, which is known for its accurate quantification of neurobehavioral abnormalities in dementia patients. It evaluates a wide range of psychopathology symptoms. As it is completed by the carer, it helps collect a wider array of information on the most pertinent problems of the patient in day-to-day life. Also, this is done without encroaching into the time of the clinician. The CBI has been found to be of use not only in dementia but also in Parkinson's disease, and parkinsonism is well known to occur in iNPH $[11,14]$. Because of these reasons, we chose the CBI. We used a comprehensive scale and hence we can be sure that all neuropsychiatric manifestations have been captured. 
All our patients had one or more neuropsychiatric symptoms; however, the severity of the impairment of individual items appeared to be mild. Earlier studies have shown that up to $85 \%$ of patients with advanced [15] dementia will have neuropsychiatric manifestations. Our study shows that iNPH is no exception to this rule.

Apathy is an important neuropsychiatric abnormality in iNPH. It has been postulated that apathy contributes to gait abnormality in iNPH [16]. Peterson et al. [17] found that a greater amount of ventriculomegaly was associated with greater apathy. In his study, they could show that improvement in apathy after shunt surgery led to improvement in the MMSE score. They postulated that apathy may be indicative of more subcortical atrophy and hence may relate to functional outcome after surgery. In our study, apathy was also found to be an important neuropsychiatric abnormality. Lack of motivation was the main neuropsychiatric manifestation in our patients, both in terms of number of patients involved as well as severity of involvement. Lack of motivation is a manifestation of apathy. Other features of apathy include abulia, decreased empathy, and lack of emotional involvement [18]. Among the 5 important frontal subcortical circuits, motivation is mediated by the anterior cingulate circuit $[19,20]$. Hypoperfusion in the anterior cingulate cortex and thalamus has already been described in iNPH patients [21-23]. Our findings support this assumption. The present study was compared with the study by Kito et al. [6]. As the NPI was used as the scale for behavioral abnormalities in their study unlike our study where the CBI was used, the results were not easily comparable. However, their results were comparable to our study as the abnormality seen in the majority of patients was apathy, which was similar to the abnormal motivation seen in our patients. Studies have shown that the ventromedial superior frontal gyrus lesion is the most specific lesion associated with apathy. The same structure is also critical in motivation [24]. Hence, both studies may be pointing towards the same structural lesion in iNPH. In the cohort of Kito et al. [6], apathy was found to be mild and was partly attributed to the less cognitively advanced stage of the disease (MMSE score 19). However, in our study, in spite of the cognitively advanced state, apathy was mild, thereby indicating that the degree of apathy may not be really related to the degree of cognitive impairment.

Sleep disturbance was one of the major abnormalities in our cohort, seen in $85 \%$ of patients [25]. Sleep abnormality was seen in the form of disturbed sleep during the night and increased sleep during daytime. This was important in terms of the number of patients involved as well as in terms of severity as reflected by the percentage of the maximum score. Detailed assessment was not done in the study. Isolated reports on sleep abnormalities in iNPH are available; however, this aspect has not been considered seriously [26]. In the series of Kito et al. [6], this has not been mentioned probably because the NPI used did not capture this abnormality. Sleeping problems in NPH need a more detailed evaluation in a larger number of patients before any firm conclusions can be made.

Considering the number of patients affected, impairment of day-to-day skill was the second commonest neuropsychiatry symptom. However, the mean CBI score in this regard was low, thereby indicating only mild impairment.

In one of the recently published studies on cognitive abnormalities in patients with iNPH by the same authors, it was found that cognitive impairment in advanced iNPH was similar to that of advanced AD patients [7]. Hence, we decided to compare the neurobehavioral abnormalities of iNPH with those of AD. When compared with AD, the CBI score and subscores were higher for iNPH even though cognitively AD patients showed more advanced disease. This may be because neurobehavioral manifestations in dementia are not a direct correlate of cognitive impairment. Also, no direct correlation was seen between cognitive scores and neurobehavioral scores. From the present study, it appears that the pattern of neurobehavioral impairment in AD and iNPH are more or less similar; however, the severity is less in iNPH. However, motivation appears more involved in iNPH, a finding which needs to be repli- 
cated in larger studies. Apathy has been mentioned as the most common behavioral and psychological symptom in dementia; however, our study shows that apathy may be more prominent in iNPH than in AD even though the difference in motivation scores did not reach statistical significance.

Three patients presented with psychiatric illness, not dementia. This is an uncommon presentation of iNPH. Reports on psychiatric presentation of iNPH are sparse and consist of mostly case reports $[27,28]$. In one of the largest series evaluating psychiatric manifestations of iNPH, Oliveira et al. [29] concluded that iNPH can present as psychiatric syndromes, which need to be considered in the evaluation of psychiatric patients. In their series, 25 out of 35 patients presented with psychiatric syndromes. In their series, all patients had undergone psychiatric evaluation. In our series, this aspect was not looked into seriously and psychiatrist evaluation was done only in situations where it was inevitable. However, this aspect of the disease needs more serious evaluation before firm conclusions can be made.

One of the major limits of the study is that the sample size is small and for the same reason results are not generalizable before replication in larger studies. Many of the observations would have probably reached statistical significance if the sample size was larger. We chose to use the abridged version of the CBI, which means that some of the minor behavioral abnormalities might have been missed. As the patients were having significant cognitive impairment at the time of evaluation, the neuropsychiatric abnormalities seen in earlier stages of the disease might have been missed.

It can be concluded that neurobehavioral abnormalities are common in patients with cognitively advanced NPH. However, the intensity of involvement appeared to be less when compared to AD. Apathy appears to be the most common domain of impairment. Sleep abnormality is a neurobehavioral abnormality which was commonly seen in this cohort and needs further evaluation.

\section{Acknowledgement}

We acknowledge Dr. Zailu and Dr. Gopika Gopinathan for editorial assistance.

\section{Disclosure Statement}

The authors declare that there are no conflicts of interest.

\section{Funding Sources}

The authors received no funding for this study.

\section{References}

1 Rabins PV, Mace NL, Lucas MJ. The impact of dementia on the family. JAMA. 1982 Jul;248(3):333-5.

2 Lyketsos CG, Lopez O, Jones B, Fitzpatrick AL, Breitner J, DeKosky S. Prevalence of neuropsychiatric symptoms in dementia and mild cognitive impairment: results from the cardiovascular health study. JAMA. 2002 Sep; 288(12):1475-83.

3 Lyketsos CG, Carrillo MC, Ryan JM, Khachaturian AS, Trzepacz P, Amatniek J, et al. Neuropsychiatric symptoms in Alzheimer's disease. Alzheimers Dement. 2011 Sep;7(5):532-9.

4 Larsson A, Wikkelsö C, Bilting M, Stephensen H. Clinical parameters in 74 consecutive patients shunt operated for normal pressure hydrocephalus. Acta Neurol Scand. 1991 Dec;84(6):475-82. 
5 Lindqvist G, Andersson H, Bilting M, Blomstrand C, Malmgren H, Wikkelsø C. Normal pressure hydrocephalus: psychiatric findings before and after shunt operation classified in a new diagnostic system for organic psychiatry. Acta Psychiatr Scand Suppl. 1993;373:18-32.

6 Kito Y, Kazui H, Kubo Y, Yoshida T, Takaya M, Wada T, et al. Neuropsychiatric symptoms in patients with idiopathic normal pressure hydrocephalus. Behav Neurol. 2009;21(3):165-74.

7 Mathew R, Pavithran S. Cognition in advanced normal pressure hydrocephalus: A pilot study from South India. Neurol India. 2017 Jul-Aug;65(4):729-31.

8 Relkin N, Marmarou A, Klinge P, Bergsneider M, Black PM. Diagnosing idiopathic normal-pressure hydrocephalus. Neurosurgery. 2005 Sep;57(3 Suppl):S4-16.

9 McKhann G, Drachman D, Folstein M, Katzman R, Price D, Stadlan EM. Clinical diagnosis of Alzheimer's disease: report of the NINCDS-ADRDA Work Group under the auspices of Department of Health and Human Services Task Force on Alzheimer's Disease. Neurology. 1984 Jul;34(7):939-44.

10 Bozeat S, Gregory CA, Ralph MA, Hodges JR. Which neuropsychiatric and behavioural features distinguish frontal and temporal variants of frontotemporal dementia from Alzheimer's disease? J Neurol Neurosurg Psychiatry. 2000 Aug;69(2):178-86.

11 Wedderburn C, Wear H, Brown J, Mason SJ, Barker RA, Hodges J, et al. The utility of the Cambridge Behavioural Inventory in neurodegenerative disease. J Neurol Neurosurg Psychiatry. 2008 May;79(5):500-3.

12 Finkel SI, Costa e Silva J, Cohen G, Miller S, Sartorius N. Behavioral and psychological signs and symptoms of dementia: a consensus statement on current knowledge and implications for research and treatment. Int Psychogeriatr. 1996;8(S3 Suppl 3):497-500.

13 Kales HC, Gitlin LN, Lyketsos CG. Assessment and management of behavioral and psychological symptoms of dementia. BMJ. 2015 Mar 2;350:h369.

14 Nagahama Y, Okina T, Suzuki N, Matsuda M. The Cambridge Behavioral Inventory: validation and application in a memory clinic. J Geriatr Psychiatry Neurol. 2006 Dec;19(4):220-5.

15 Kverno KS, Black BS, Blass DM, Geiger-Brown J, Rabins PV. Neuropsychiatric symptom patterns in hospiceeligible nursing home residents with advanced dementia. J Am Med Dir Assoc. 2008 Sep;9(7):509-15.

16 Allali G, Laidet M, Armand S, Saj A, Krack P, Assal F. Apathy and higher level of gait control in normal pressure hydrocephalus. Int J Psychophysiol. 2017 Sep;119:127-31.

17 Peterson KA, Housden CR, Killikelly C, DeVito EE, Keong NC, Savulich G, et al. Apathy, ventriculomegaly and neurocognitive improvement following shunt surgery in normal pressure hydrocephalus. Br J Neurosurg. 2016;30(1):38-42.

18 Moretti R, Signori R. Neural Correlates for Apathy: Frontal-Prefrontal and Parietal Cortical- Subcortical Circuits. Front Aging Neurosci. 2016 Dec;8:289.

19 Bonelli RM, Cummings JL. Frontal-subcortical circuitry and behavior. Dialogues Clin Neurosci. 2007;9(2): 141-51.

20 Cummings JL. Frontal-subcortical circuits and human behavior. Arch Neurol. 1993 Aug;50(8):873-80.

21 Takeuchi T, Goto H, Izaki K, Tamura S, Tomii M, Sasanuma J, et al. Pathophysiology of cerebral circulatory disorders in idiopathic normal pressure hydrocephalus. Neurol Med Chir (Tokyo). 2007 Jul;47(7):299-306.

22 Murakami M, Hirata Y, Kuratsu JI. Predictive assessment of shunt effectiveness in patients with idiopathic normal pressure hydrocephalus by determining regional cerebral blood flow on 3D stereotactic surface projections. Acta Neurochir (Wien). 2007 Oct;149(10):991-7.

23 Klinge PM, Brooks DJ, Samii A, Weckesser E, van den Hoff J, Fricke H, et al. Correlates of local cerebral blood flow (CBF) in normal pressure hydrocephalus patients before and after shunting - A retrospective analysis of [(15)0]H(2)O PET-CBF studies in 65 patients. Clin Neurol Neurosurg. 2008 Apr;110(4):369-75.

24 Rosen HJ, Allison SC, Schauer GF, Gorno-Tempini ML, Weiner MW, Miller BL. Neuroanatomical correlates of behavioural disorders in dementia. Brain. 2005 Nov;128(Pt 11):2612-25.

25 Simuni T, Sethi K. Nonmotor manifestations of Parkinson's disease. Ann Neurol. 2008 Dec;64(S2 Suppl 2):S65-80.

26 Munari C, Calbucci F, Benericetti E, Dallolio W, Versari P. [Restructuring the sleep of a patient with NPH (normal pressure hydrocephalus/ after ventricular CSF shunt]. Riv Neurol. 1977 May-Jun;47(3):259-66.

27 Pinner G, Johnson H, Bouman WP, Isaacs J. Psychiatric manifestations of normal-pressure hydrocephalus: a short review and unusual case. Int Psychogeriatr. 1997 Dec;9(4):465-70.

28 Groenewald E, Joska JA, Rothemeyer S. Normal-pressure hydrocephalus presenting with psychiatric symptoms. S Afr Med J. 2016 Jan;106(2):162.

29 Oliveira MF, Oliveira JR, Rotta JM, Pinto FC. Psychiatric symptoms are present in most of the patients with idiopathic normal pressure hydrocephalus. Arq Neuropsiquiatr. 2014 Jun;72(6):435-8. 